\title{
Localización Biométrica de los Puntos Motores en el Músculo Tríceps Braquial de individuos Brasileños
}

\author{
Biometric Location of Motor Points in the Brachial Triceps Muscle of Brazilian individuals
}

\author{
Lizama, R. ${ }^{1,2}$; Sousa-Rodrigues, C. F. ${ }^{3}$ \& Olave, E. ${ }^{4}$
}

\begin{abstract}
LIZAMA, R.; SOUSA-RODRIGUES, C. F. \& OLAVE, E. Localización biométrica de los puntos motores en el músculo tríceps braquial de individuos Brasileños. Int. J. Morphol., 37(1):379-384, 2019.

RESUMEN: El músculo tríceps braquial, es el motor primario para el movimiento de extensión de codo, por lo que una lesión que afecte su función perjudicaría enormemente la calidad de vida de los afectados. El conocimiento de su inervación y la localización biométrica de sus puntos motores, es una herramienta útil en terapias de electro estimulación muscular. El objetivo del estudio fue determinar el número y localización de los puntos motores de este músculo. Para ello, se utilizaron 30 miembros superiores de individuos brasileños, a los cuales se les realizó una disección detallada del compartimiento posterior del brazo. Se registró el número de ramos, puntos motores y localización biométrica de cada uno de los ramos destinados a las cabezas del músculo triceps braquial. Se utilizó como punto de referencia una Línea biepicondilar, trazada entre los epicóndilos humerales. En todos los casos este músculo estaba inervado por el nervio radial. El promedio de puntos motores (PM) para la cabeza larga del músculo (CL) fue de 3,9 $\pm 1,4 ; 4,8 \pm 1,2$ para la cabeza medial (CM) y 4,1 $\pm 1,4$ para la cabeza lateral (CLat). Los puntos motores se concentraron preferentemente en el tercio medio del brazo, tanto a nivel general, como también por cada cabeza. Los datos biométricos aportados complementarán el conocimiento de la inervación de este músculo y favorecerá una mejor comprensión y elección de tratamientos frente a una patología.
\end{abstract}

PALABRAS ClAVE: 1. Anatomía 2. Tríceps braquial 3. Inervación 4. Puntos motores

\section{INTRODUCCIÓN}

El músculo tríceps braquial (MTB) se ubica en el compartimiento posterior del brazo, y en sus orígenes consta de tres cabezas independientes, la cabeza larga (CL) la cabeza medial (CM) y la cabeza lateral (CLat) (GarcíaPorrero \& Hurlé, 2005; Pró, 2012).

Textos clásicos de anatomía señalan que su inervación proviene del nervio radial (NR) (Orts Llorca, 1959; Testut \& Latarjet, 1972; Rouvière \& Delmas, 2005), aunque también existen estudios donde se informa la participación de otros nervios en la inervación de este músculo (de Sèze et al., 2004; Pascual-Font et al., 2013; Molina et al., 2017). Es el músculo motor primario para el movimiento de extensión de codo y no puede ser sustituido cuando está paralizado (Orts Llorca; Prikc' et al., 2018), por lo que una lesión nerviosa, influye considerablemente en la funcionalidad del miembro superior, perjudicando enormemente la calidad de vida de los afectados. La aplicación de técnicas, tanto de estimulación en caso de un músculo atrofiado o de inhibi- ción en caso de un músculo rígido o espástico, son de importancia en la terapia física (Riveros et al., 2018).

En este contexto, conocer la inervación motora y sus variaciones es de importancia para diagnosticar correctamente la lesión de algún nervio y la elección de un adecuado tratamiento (Olave et al., 2002). Conocer además, la ubicación de los puntos motores representa un referente anatomo-funcional importante para el uso de diferentes herramientas. (Ballesteros, 2015; Yang et al., 2017).

Funcionalmente, un punto motor se define como el área en la que un estímulo eléctrico de intensidad mínima y corta duración provoca una contracción muscular (Childers, 2003; Lapatki et al., 2011), en cambio, desde el punto de vista morfológico, el punto motor (PM) se define como el sitio en el que el ramo motor de un nervio ingresa al vientre muscular (Ballesteros). Esta última definición se acerca más al objetivo real al momento de realizar terapias con toxina

\footnotetext{
${ }^{1}$ Departamento de Ciencias Morfológicas, Facultad de Medicina y Ciencia, Universidad San Sebastián, General Lagos 1163, Valdivia 5090000, Chile.

${ }^{2}$ Programa de Magíster en Ciencias mención Morfología, Universidad de La Frontera, Temuco, Chile.

${ }^{3}$ Universidad Estadual de Ciencias da Saude de Alagoas, Maceió, Brasil.

${ }^{4}$ Facultad de Medicina, Universidad de La Frontera, Temuco, Chile.
} 
botulínica en pacientes con espasticidad (Tecglen et al., 2014). Lapatki et al., han establecido que si se inyecta cerca de la placa motora, se puede utilizar una dosis menor del fármaco obteniendo un beneficio superior, misma situación aplica en el tratamiento de esta espasticidad a través de la neurolisis (Lam et al., 2015; Yang et al.).

A pesar de la importancia de este músculo, y de lo complejo de su lesión, existe escasez de estudios cadavéricos que describan la localización de sus puntos motores, más aún si buscamos investigaciones realizadas en este continente.

El objetivo de este estudio fue determinar la localización biométrica de los puntos motores del tríceps braquial con respecto a una referencia ósea.

\section{MATERIAL Y MÉTODO}

En el presente estudio se utilizaron 30 miembros superiores, 16 derechos y 14 izquierdos (28 Masculinos; $2 \mathrm{Fe}$ meninos), de individuos adultos Brasileños, del Laboratorio de Anatomía de la Facultad de Medicina de la Universidad Estadual de Ciencias da Saúde de Alagoas, Maceió, Brasil. Todos los cadáveres se encontraban fijados bajo inmersión en una solución con formaldehído al $10 \%$.

La presente investigación se realizó con una metodología de carácter cuantitativo, no experimental, transversal y descriptivo, en la cual se procedió a realizar una detallada disección del compartimiento posterior del brazo con material quirúrgico "ad-hoc".

Se midió y registró la longitud del brazo tomando como referencias el punto más prominente del acromion y una línea trazada entre los puntos más prominentes de los epicóndilos humerales, la línea biepicondilar (LBE). A continuación se llevó a cabo la disección del compartimiento posterior del brazo, identificando el trayecto y los ramos motores del nervio radial para el músculo tríceps braquial. Luego de ser identificados estos ramos, se registró su número, punto motor (PM), tanto para la totalidad del músculo como para cada una de las tres cabezas que lo conforma.

Adicionalmente se registró la distancia entre la LBE y los puntos motores identificados. A continuación y con la ayuda de un goniómetro y una plomada de medición se representó una línea vertical que quedara dispuesta perpendicular a la LBE, se registró la distancia de esta vertical hasta el punto motor. Se enumeraron los puntos motores de proximal a distal, por lo tanto, el PM1 es el que se encontró en el punto proximal más alejado de la LBE. Para los casos en los cuales se identificaron dos puntos motores a la misma distancia se denominaron en el sentido medial a lateral (Fig. 1).

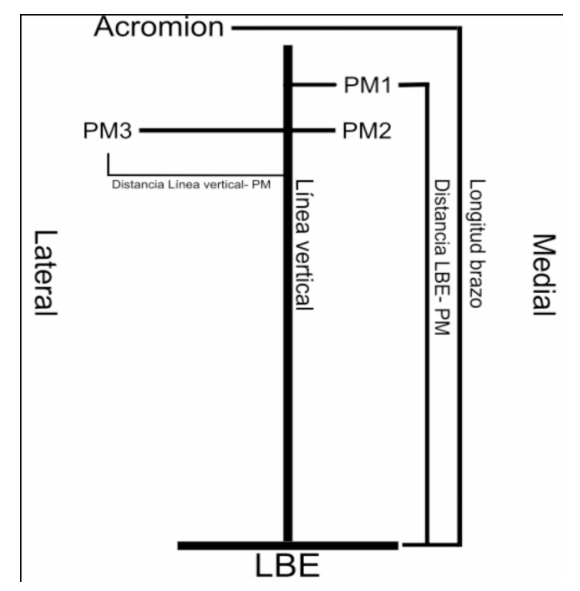

Fig. 1. Esquema que muestra las mediciones tomadas y los puntos de referencia en un brazo izquierdo. LBE: Línea biepicondilar; PM: Punto motor

Las medidas fueron tomadas con un caliper digital marca Mitutoyo ${ }^{\circledR}$ de $0,01 \mathrm{~mm}$ de precisión y se fotografiaron las muestras con una cámara digital marca Fujifilm FinePix S2980. Una vez registradas estas mediciones, se dividió la longitud del brazo (distancia LBE-acromion) en tercios proximal, medio y distal. En base a ello se contabilizó el número de PM según esta distribución.

El analisis estadistico se realizó con el programa SPSS versión 20.0, el cual incluyó estadisticos descriptivos, prueba de normalidad ( Shapiro wilk) y la prueba estadística U de Mann- Whitney para comparar las medianas de ramos y total de puntos motores según lateralidad del miembro.

\section{RESULTADOS}

En todos los casos la inervación provino del NR, el cual ingresó a la zona posterior del músculo tríceps braquial a través del intervalo triangular, el cual está formado por el margen inferior del músculo redondo mayor y el margen lateral de la CL del MTB. El NR emitió entre tres y siete ramos motores para el MTB, con un promedio de 4,7 $\pm 1,1$. Del total de ramos emitidos, el NR emitió 3 ramos (13\%), 4 ramos ( $37 \%), 5$ ramos $(23 \%), 6$ ramos $(23 \%)$ y 7 ramos (3 $\%)$. En todos los brazos estos ramos se dividieron en ramos secundarios, los cuales ingresaron al vientre muscular de alguna de las cabezas del MTB (Fig. 2). Con respecto al 


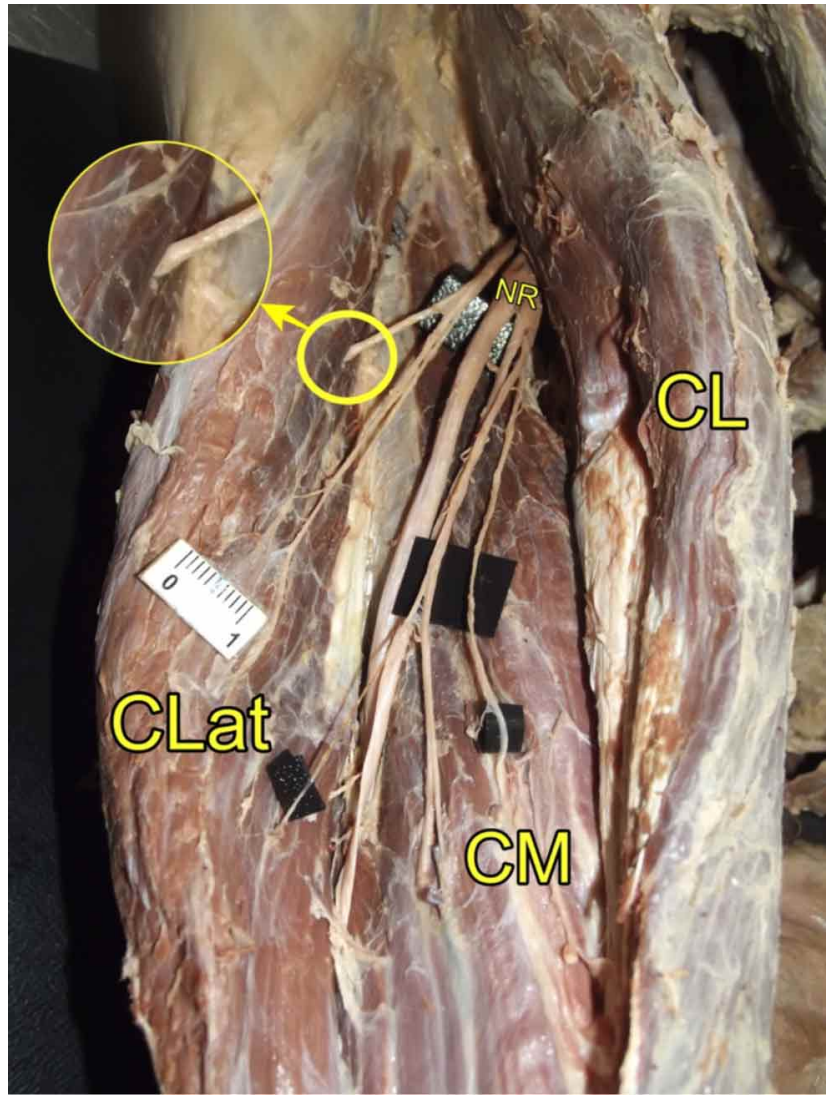

Fig. 2. Vista posterior brazo izquierdo, NR en el compartimiento posterior del brazo entregando ramos a las cabezas del MTB. NR: Nervio radial; CL: Cabeza larga; CM: Cabeza medial; CLat: Cabeza lateral; Círculo amarillo: Punto motor.

total de ramos según lateralidad del miembro superior, no existieron diferencias significativas $(\mathrm{p}=0,448)$.

El número total de PM para el MTB, varió entre 9 y 19. La presencia de $14 \mathrm{PM}$ se observó en $27 \%$ de los casos, $10 \mathrm{PM}$ en $20 \%, 9$ PM en $13 \%$ y 13 PM en $10 \%$. En el $30 \%$ restante el MTB presentó 11 (7\%), $12(3 \%), 15$ (7\%), 16 (3\%), 17 (3\%) y $19(7 \%)$ PM. No existen diferencias estadísticas significativas en el total de PM entre los brazos derecho e izquierdo $(\mathrm{p}=0,355)$.
En todos los casos y considerando las tres cabezas del MTB, la mayor cantidad de PM se ubicó en el tercio medio del brazo, con un promedio de 9,3 $\pm 2,4 \mathrm{PM}$.

Por su parte, los tercios proximal y distal presentaron un promedio de $1,4 \pm 1,5$ y $2,0 \pm 2,1 \mathrm{PM}$. Al dividir el tercio medio del brazo en mitad proximal y distal, se observó variabilidad en cuanto a la distribución de los PM para cada cabeza del MTB; lo anterior se encuentra resumido en la Tabla I.

Para la CL del MTB, el NR entregó un ramo en el 83 $\%$ de los casos y dos ramos en el $17 \%$ restante. En ambas situaciones el NR emitió ramos para la CL antes de ingresar al compartimiento posterior del brazo por el intervalo triangular (Fig.3). Con respecto a los PM, estos variaron entre dos y ocho, con un promedio de 3,9 $\pm 1,4$. En el $27 \%$ de los casos,

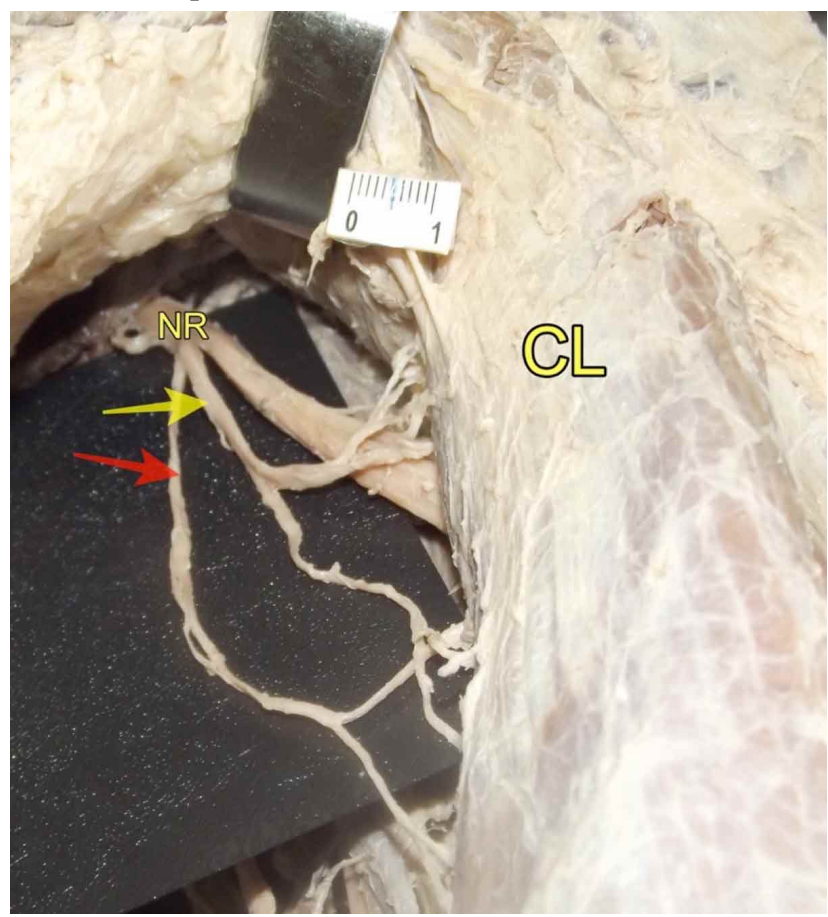

Fig. 3. NR antes de ingresar al compartimiento posterior del brazo. NR: Nervio radial; Flecha amarilla: Ramo para la cabeza larga; Flecha roja: Ramo para la cabeza medial.

Tabla I. Distribución puntos motores (PM) del músculo tríceps braquial en el tercio medio del brazo dividido en mitad proximal y distal.

\begin{tabular}{|c|c|c|c|c|c|c|c|c|c|}
\hline \multicolumn{4}{|c|}{ Anex os Cabeza larga } & \multicolumn{3}{|c|}{ Cabeza medial } & \multicolumn{3}{|c|}{ Cabeza lateral } \\
\hline PM & Casos & Proximal & Distal & Casos & Proximal & Distal & Casos & Proximal & distal \\
\hline 1 & $12 / 30$ & $100 \%$ & $0 \%$ & $29 / 30$ & $69 \%$ & $31 \%$ & $21 / 30$ & $90 \%$ & $10 \%$ \\
\hline 2 & $21 / 30$ & $100 \%$ & $0 \%$ & $27 / 30$ & $19 \%$ & $81 \%$ & $28 / 30$ & $50 \%$ & $50 \%$ \\
\hline 3 & $24 / 30$ & $100 \%$ & $0 \%$ & $23 / 30$ & $3 \%$ & $97 \%$ & $24 / 30$ & $46 \%$ & $54 \%$ \\
\hline 4 & $17 / 30$ & $88 \%$ & $12 \%$ & $12 / 30$ & $0 \%$ & $100 \%$ & $17 / 30$ & $29 \%$ & $71 \%$ \\
\hline 5 & $9 / 30$ & $67 \%$ & $33 \%$ & $3 / 30$ & $0 \%$ & $100 \%$ & $14 / 30$ & $0 \%$ & $100 \%$ \\
\hline 6 & $3 / 30$ & $33 \%$ & $67 \%$ & $2 / 30$ & $0 \%$ & $100 \%$ & $3 / 30$ & $0 \%$ & $100 \%$ \\
\hline 7 & $1 / 30$ & $100 \%$ & $0 \%$ & $0 / 30$ & - & - & $1 / 30$ & $0 \%$ & $100 \%$ \\
\hline 8 & $1 / 30$ & $0 \%$ & $100 \%$ & $0 / 30$ & - & - & - & - & - \\
\hline
\end{tabular}




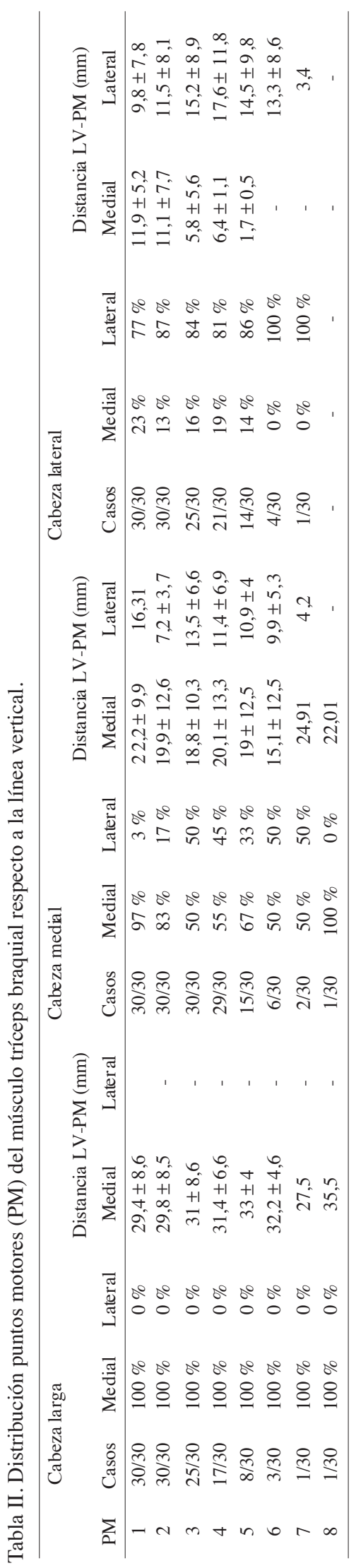

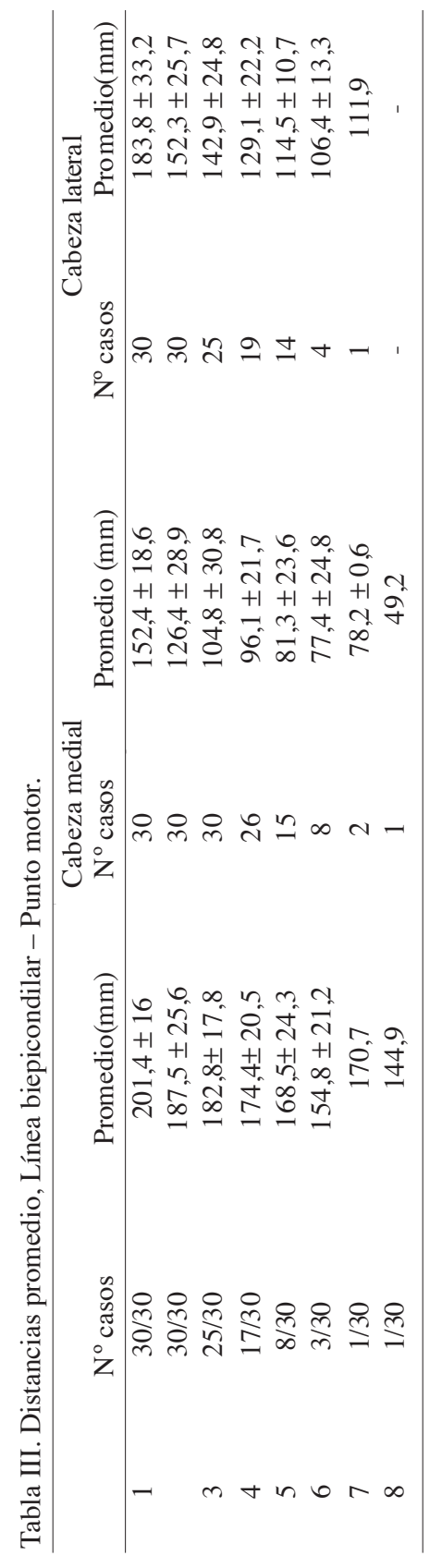

la CL presentó cuatro PM, en el $27 \%$ se observaron tres PM, en el $20 \%$ se presentaron cinco PM y en el $17 \%$, sólo 2 PM. En el $9 \%$ restante la CL presentó $6(6 \%)$ y $8(3 \%)$ PM. El $100 \%$ de los PM para la $\mathrm{CL}$, se encontraban en una posición medial con respecto a la línea vertical (Tabla II).

Para la CM el NR emitió dos ramos en el $73 \%$ de los casos, tres ramos en el $17 \%$ y un ramo en el $10 \%$. La cantidad de PM para esta cabeza varió entre tres y ocho, con un promedio de $4,8 \pm$ 1,2 . En el $40 \%$ de los casos la CM presentó cuatro PM; en el $23 \%$ cinco PM y en el $20 \%$, seis PM. En el $17 \%$ restante la CM presentó tres $(10 \%)$, siete $(3,3 \%)$ y ocho $(3,3 \%)$ PM.

Se observó variabilidad en cuanto a la ubicación del PM respecto a la línea vertical dispuesta en forma perpendicular al LBE. En algunos casos estos se ubicaron lateral, aunque en un mayor porcentaje los PM se ubican medial a la línea vertical. Estos datos quedaron resumidos en la Tabla II.

Para la CLat el NR emitió hasta tres ramos, un ramo en el $43 \%$ de los casos, dos en el $30 \%$, tres en el $13 \%$ y no emitió ningún ramo directo en el 13 $\%$ restante. La cantidad de PM para esta cabeza varió entre dos y siete, con un promedio de 4,1 $\pm 1,4$ PM. La CLat presentó cinco PM en el $33 \%$ de los casos, cuatro PM en el $17 \%$, tres PM en el $20 \%$ y dos PM en $17 \%$. Enel $13 \%$ restante, la CLat presentó seis $(10 \%)$ y siete $(3 \%)$ PM. Respecto a la localización de estos PM en relación a la línea vertical, esta cabeza también presentó variabilidad, destacando que la mayoría de sus PM fueron ubicados en un punto lateral a la mencionada línea de referencia. La localización de estos PM respecto a la línea vertical se encuentran resumidos en la Tabla II.

En lo que respecta a las distancias entre la LBE y los PM de cada una de las cabezas del MTB, estas se encuentran resumidas en la Tabla III. 


\section{DISCUSIÓN}

Textos clásicos de anatomía señalan que el MTB esta inervado por el NR en toda su extensión (Orts Llorca; Testut \& Latarjet; Rouvière \& Delmas). Esta situación fue evidenciada en la totalidad de los casos analizados en nuestra investigación. Existen también, autores que señalan la presencia de otros nervios como el ulnar y el axilar en su inervación (Pascual-Font et al.; Molina et al.; Erhardt et al., 2017). Por su parte, Bertelli et al. (2007) y Torres et al. (2018), observaron que la inervación del MTB provenía exclusivamente del NR, información que coincide con lo observado en este estudio.

En nuestro estudio se observó que el número de ramos para el MTB varió entre tres y siete, de los cuales lo que más preponderó fue la presencia de cuatro ramos (37 $\%)$. Al respecto, Torres et al., encontraron entre dos y cinco, diferente de nuestros hallazgos; sin embargo, en ambos estudios el número de ramos que se presentó con mayor frecuencia fue de cuatro.

Con respecto al número de ramos motores para cada cabeza, Bertelli et al., observaron un ramo para la CL, resultado con el que concordamos ya que el 83,3\% de nuestros casos tuvieron ese ramo único. Similar situación fue relatada por Torres et al. en más del del $50 \%$ de sus casos estudiados.

Dos ramos motores para la CLat fueron observados por Bertelli et al., ramos que sólo observamos en un $30 \%$, ya que la presencia de un ramo para la cabeza lateral fue lo más frecuente $(43,3 \%)$. Similar situación fue observada por Torres et al. quienes observaron un ramo para la CLat en más del $50 \%$ de sus muestras. Por su parte, Cho et al. (2013) señalaron que el número máximo de ramos para la CLat fue de cuatro, diferente de nuestros resultados, donde el número fue de tres. Estos mismos autores observaron que la CLat presentó la mayor cantidad de ramos, mientras que en nuestra serie, la cabeza muscular que más presentó ramos fue la CM.

En el presente estudio, el número total de PM para el MTB varió entre 9 y 19, semejante a lo encontrado por Torres et al. Quienes observaron entre 8 y 20 PM (48 muestras). Por su parte, los mismos autores encontraron que la CL fue la que presentó mayor cantidad de PM, mientras que nuestros resultados muestran que la cabeza que más presentó $\mathrm{PM}$ fue la $\mathrm{CM}$ con un promedio de 4,8 $\pm 1,2$.

La mayor cantidad de ramos y puntos motores de la cabeza medial, se podría explicar a través de la biomecánica de la extensión de codo, donde se sabe que la contracción de esta cabeza es primordial para producir este movimiento, siendo la que más participa y está activa en todas las posiciones para este movimiento (Hamill et al., 2015).

La ubicación de los PM es un dato importante para el clínico, ya que es esencial para un adecuado uso de terapia de estimulación eléctrica (Torres et al.). Así mismo, la ubicación de los PM con respecto a una línea vertical, entrega al clínico un dato relevante y de mayor precisión, generando una localización no tan sólo a lo largo del brazo, sino que también a lo ancho, lo cual facilita una localización más precisa de los PM, al momento de realizar los diferentes tratamientos.

La localización de los PM a partir de una referencia ósea (LBE), es un dato que entrega mayor utilidad a los clínicos, considerando que no siempre se cuenta con equipos de imagen al momento de realizar procedimientos de electro diagnostico o terapias de electro estimulación. Además, los epicóndilos humerales son reparos óseos fáciles de palpar y de encontrar, por lo que los datos entregados ayudarán a una fácil ubicación de los PM en el vivo, aún sin tener acceso a equipos de imagen.

No hubo comparación entre sexos por causa de tener sólo dos miembros superiores del sexo femenino.

Si bien es cierto, ya se han hecho algunos estudios en los cuales se describe la localización de los puntos motores en diferentes músculos, (Olave et al., 2002, 2009, 2012; Olave \& Braga, 2003; Méndez et al., 2009; Riveros et al., 2016; Torres et al.), el número limitado de estudios referentes al tríceps braquial en muestras cadavéricas posiciona a esta investigación como un interesante aporte de datos biométricos, que contribuirán al conocimiento de la inervación de este músculo, y favorecerá una mejor comprensión y elección de tratamientos frente a una patología.

LIZAMA, R.; SOUSA-RODRIGUES, C. F. \& OLAVE, E. Biometric Location of Motor Points in the Brachial Triceps Muscle of Brazilian individuals. Int. J. Morphol., 37(1):379-384, 2019.

SUMMARY: The triceps brachii muscle is the primary motor for elbow extension movement, so a lesion that affects its function would greatly harm the quality of life of those affected. The knowledge of its innervation and the biometric localization of its motor points is a useful tool in electro-stimulation muscular therapies. The objective of the study was to determine the number of branches and location of the motor points of this muscle. To this 
end, 30 superior members of Brazilian individuals were used, to whom a detailed dissection of the posterior compartment of the arm was performed. The number of branches, motor points and biometric location of each of the branches destined for the three heads of the brachial triceps muscle was recorded. A biepicondilar line, traced between the humeral epicondyles, was used as a reference point. In all cases, this muscle was innervated by the radial nerve. The average motor points for the long head of the muscle $(\mathrm{LH})$ was $3.9+1.4$; for the medial head $(\mathrm{MH})$ was $4.8+$ 1.2 and for the lateral head $(\mathrm{LatH})$ was $4.1+1.4$. The motor points were concentrated mainly in the middle third of the arm, both at a general level, and also for each head. The biometric data provided will complement the knowledge of the innervation of this muscle and will favor a better understanding and choice of treatments for a pathology.

KEY WORDS: Anatomy, Brachial Triceps muscle, Innervation, Motor points.

\section{REFERENCIAS BIBLIOGRÁFICAS}

Ballesteros, L. E. Los puntos motores: una expresión morfológica con amplias aplicaciones clínicas que no se ha estudiado suficientemente. Rev. Argent. Anat. Clin., 7(2):86-7, 2015.

Bertelli, J. A.; Santos, M. A.; Kechele, P. R.; Ghizoni, M. F. \& Duarte, H. Triceps motor nerve branches as a donor or receiver in nerve transfers. Neurosugery, 61(5 Suppl. 2):333-9, 2007.

Childers, M. K. The importance of electromyographic guidance and electrical stimulation for injection of botulinum toxin. Phys. Med. Rehabil. Clin. N. Am., 14(4):781-92, 2003.

Cho, H.; Lee, H. Y.; Gil, Y. C.; Choi, Y. R. \& Yang, H. J. Topographical anatomy of the radial nerve and its muscular branches related to surface landmarks. Clin. Anat., 26(7):262-8, 2013.

de Sèze, M. P.; Rezzouk, J.; de Sèze, M.; Uzel, M.; Lavignolle, B.; Midy, D. \& Durandeau, A. Does the motor branch of the long head of the triceps brachii arise from the radial nerve? An anatomic and electromyographic study. Surg. Radiol. Anat., 26(6):459-61, 2004.

Erhardt, A. J. \& Futterman, B. Variations in the innervation of the long head of the triceps brachii: a cadaveric investigation. Clin. Orthop. Relat. Res., 472(1):247-50, 2017.

García-Porrero, J. \& Hurlé, J. Anatomía Humana. Madrid, McGraw-Hill, 2005. pp.191-3.

Hamill, J.; Knutzen, K. \& Derrick, T. Biomechanica. Basis of Human Movement. $4^{\mathrm{a}}$ ed. Philadelphia, Wolters Kluwer, 2015. pp.152-3.

Lam, K.; Wong, D.; Tam, C. K.; Wah, S. H.; Myint, M. W.; Yu, T. K.; So, K. K.; Cheung, G.; Au, K. M.; Fu, M. H.; Wu, Y. M. \& Kng, C. P. Ultrasound and electrical stimulator-guided obturator nerve block with phenol in the treatment of hip adductor spasticity in long-term care patients: a randomized, triple blind, placebo controlled study. J. Am. Med. Dir. Assoc., 16(3):238-46, 2015.

Lapatki, B. G.; van Dijk, J. P.; van de Warrenburg, B. P. \& Zwarts, M. J. Botulinum toxin has an increased effect when targeted toward the muscle's endplate zone: a high-density surface EMG guided study. Clin. Neurophysiol., 122(8):1611-6, 2011.

Méndez, R. G.; Coronado, G. R. \& Suazo, G. I. Anatomical study of the fibularis longus muscle motor points and electrical stimulation therapy application. Int. J. Morphol., 27(3):699-703, 2009.

Molina, C. R.; Díaz, I.; Taunton, M. J.; Flores, E.; Rosas, C. \& Letelier, R. Innervation of the long head of triceps brachii muscle. Int. J. Morphol., 35(2):442-4, 2017.
Olave, E. \& Braga, M. T. T. Innervation of the semitendinous muscle, biometry of the branches and location of its motor points. Int. J. Morphol., 21(2):161-5, 2003.

Olave, E.; Gabrielli, C. \& Braga, M. T. T. Innervation patterns and biometric localization of the motor points of the brachial biceps muscle in man. Int. J. Morphol., 27(2):495-501, 2009.

Olave, E.; Gabrielli, C.; Braga, M. T. T. \& del Sol, M. Biometric aspects of the motor branches of the musculocutaneous nerve to the brachial muscle. Rev. Chil. Anat., 20(2):231-6, 2002.

Olave, E.; Retamal, P.; Galaz, C. \& Cruzat, C. Innervation and localization of the motor points of triceps surae muscle. Int. J. Morphol., 30(3):105660, 2012.

Orts Llorca, F. Anatomía Humana. Vol. 2. Barcelona, Científica-Médica, 1959. pp.191-7.

Pascual-Font, A.; Vasquez, T.; Marco, F.; Sañundo, J. R. \& RodriguezNiedenführ, M. Ulnar nerve innervation of the triceps muscle: real or apparent? An anatomic study. Clin. Orthop. Relat. Res., 471(6):188793, 2013.

Prikc', A.; Viveen, J.; The, B.; Van Bergen, C. J.; Koenraandt, K. L. \& Eygendaal, D. Comparison of isometric triceps brachii force measurement in different elbow positions. J. Orthop. Surg. (Hong Kong), 26(2):2309499018783907, 2018.

Pró, E. A. Anatomía Clínica. $2^{\mathrm{a}}$ ed. Buenos Aires, Médica Panamericana, 2012. pp.790-1.

Riveros, A.; Sousa-Rodrigues, C. F. \& Olave, E. Origin and distribution of the motor branches for the superficial muscles of the anterior region of the forearm in Brazilian individuals. Int. J. Morphol., 34(1):356-64, 2016.

Riveros, A.; Sousa-Rodrigues. C. F. \& Olave, E. Innervation of the superficial flexor digitorum muscle. Int. J. Morphol., 36(2):768-77, 2018.

Rouvière, H.; Delmas, A. \& Delmas, V. Anatomía Humana. Descriptiva, Topográfica y Funcional. Vol. 3. 11 a ed. Barcelona, Masson, 2005.

Tecglen, C.; Aguilar, M.; Bascones, LM.; Biurrun, J.; Cayo, P.; Chacartegui, M.; Dominguez, L.; Folgado, I.; Fuentes, V.; García, M.; Gavilán, B.; Lerma, S.; Martinez, I.; Martinez,J.; Martinez, J.; Martorell, V.; Muñoz, V.; Muyor,J.; Pascual, M.; Pereira, A.; Ramiro, J.; Sebastiá, A.; Velasco, M. \& Villén, S. Guía para las Personas que Conviven con la Espasticidad. Madrid, Imprenta Nacional de la AEBOE, 2014.

Testut, L. \& Latarjet, A. Tratado de Anatomía Humana. Vol. 1. $9^{\mathrm{a}}$ ed. Barcelona, Salvat, 1972.

Torres, E. A.; Ballesteros, L. E. \& Forero, P. L. Biometry of the Brachial Triceps Muscle and its Motor Points. A Direct Anatomical Study in a Sample of Colombian Population. Int. J. Morphol., 36(3):948-54, 2018.

Yang, S.; Hu, S; Tian, X. \& Hu, S. A novel method for localizing nerve entry points during spasticity treatment. Int. J. Morphol., 35(3):799803, 2017.

\author{
Dirección para correspondencia: \\ Dr. Enrique Olave \\ Facultad de Medicina \\ Universidad de La Frontera \\ Casilla 54-D, Temuco \\ CHILE
}

Email: enrique.olave@ufrontera.cl

Recibido : 27-10-2018

Aceptado : 14-12-2018 